\title{
fullsibQTL: an R package for QTL mapping in biparental populations of outcrossing species
}

Rodrigo Gazaffi ${ }^{1, \dagger}$, Rodrigo R. Amadeu ${ }^{2, \dagger}$, Marcelo Mollinari ${ }^{3}$, João R. B. F.

Rosa $^{4}$, Cristiane H. Taniguti ${ }^{5}$, Gabriel R. A. Margarido ${ }^{5}$, and Antonio A. F. Garcia $^{5, *}$

${ }^{1}$ Departamento de Biotecnologia e Produção Vegetal e Animal, Centro de Ciências Agrárias, Universidade Federal de São Carlos, Araras, São Paulo, Brazil; ${ }^{2}$ Blueberry Breeding \& Genomics Lab, Horticultural Sciences Department, University of Florida, Gainesville, FL, USA; ${ }^{3}$ Bioinformatics Research Center, North Carolina State University, Raleigh, NC, USA; ${ }^{4}$ Centro de Tecnologia Canavieira (CTC), Piracicaba, $S P$; and ${ }^{5}$ Departamento de Genética, Escola Superior de Agricultura Luiz de Queiroz, Universidade de São Paulo, Piracicaba, São Paulo, Brazil.

Correspondence*:

Departamento de Genética, Escola Superior de Agricultura Luiz de Queiroz, Universidade de São Paulo, Piracicaba, São Paulo, 13418-900, Brazil. augusto.garcia@usp.br

$\dagger$ : These authors have contributed equally to this work

\section{ABSTRACT}

Accurate QTL mapping in outcrossing species requires software programs which consider genetic features of these populations, such as markers with different segregation patterns and different level of information. Although the available mapping procedures to date allow inferring QTL position and effects, they are mostly not based on multilocus genetic maps. Having a QTL analysis based in such maps is crucial since they allow informative markers to propagate their information to less informative intervals of the map. We developed fullsibetL, a novel and freely available R package to perform composite interval QTL mapping considering outcrossing populations and markers with different segregation patterns. It allows to estimate QTL position, effects, segregation patterns, and linkage phase with flanking markers. Additionally, several statistical and graphical tools are implemented, for straightforward analysis and interpretations. fullsibQTL is an R open source package with $\mathrm{C}$ and $\mathrm{R}$ source code (GPLv3). It is multiplatform and can be installed from https://github.com/augusto-garcia/fullsibeTL.

Keywords: F1 population, fullsib, composite interval mapping, interval mapping, outcross

\section{INTRODUCTION}

Mapping quantitative trait loci (QTL) unveils the genetic architecture of quantitative traits (e.g. yield, fitness) and has several applications, such as breeding and evolutionary studies. In plant breeding, this information can be integrated into breeding programs with marker-assisted selection tools aiming to understand and boost such traits. Interval mapping (Lander and Botstein, 1989), composite interval mapping (Zeng, 1993, 1994), and multiple interval mapping (Kao et al., 1999) are some of the available approaches to perform QTL mapping. In populations developed from inbred (homozygous) lines, such as F2s, backcrosses and recombinant inbred lines, these mapping methods are well established and implemented in a plethora of good software, for example: Broman et al. (2003); Van Ooijen (2004); Wang et al. (2012); Meng et al. 


\section{CONCLUSION} features.

\section{FEATURES}

(2015). However, for outcrossing species without inbred lines (e.g. citrus, eucalyptus, sugarcane, loblolly pine, and rubber tree), there is a lack of freely available software programs for QTL analysis. For biparental populations derived from the cross between non-homozygous parents, it is necessary to estimate QTL number, effects and position, as well their linkage phase with markers, and segregation patterns. This effort relies on a linkage map that provides the phase of markers in both parents and their complete transmission pattern to the offspring, which is usually thoroughly achieved by using multipoint approaches.

fullsibQTL is a free R package that performs composite interval mapping in full-sib progeny (F1 population) derived from a bi-parental cross between two non-homozygous parents. It requires a previously estimated genetic map, considering markers with different segregation patterns, as described by (Wu et al., 2002b). It implements the model proposed by Gazaffi et al. (2014), allowing to estimate the number of QTLs, their position, effects, segregation patterns, and linkage phases with flanking markers. This methodology has been successfully applied in different studies (Souza et al., 2013; Margarido et al., 2015; Costa et al., 2016; Balsalobre et al., 2017; da Silva Pereira et al., 2017; Barreto et al., 2018; Conson et al., 2018; Curtolo et al., 2020; Soratto et al., 2020), but currently there is no competing software with such

fullsibeTL is a toolbox to perform QTL mapping based on composite interval mapping using the model proposed by Gazaffi et al. (2014) for outcrossing species. It considers that a multipoint genetic map was previously estimated with package oneMap, also freely available and widely used (Margarido et al., 2007, 2020). Briefly, the model has three genetics effects, one additive effect for each parent and one for the interaction of these effects (dominance). These parameters are estimated based on the maximum likelihood for mixture models, with the expectation maximization algorithm. QTL genotypes probabilities are calculated using a multipoint approach based on hidden Markov models (Wu et al., 2002a; Mollinari et al., 2009). This allows the estimation using the information of all the markers and individuals, even if there is missing data (Wu et al., 2002a).

The package also has a function to select cofactors using multiple linear regression through stepwise and information criteria (R Core Team, 2017). Permutation tests (Churchill and Doerge, 1994) for threshold determination can be implemented, combined with modifications suggested by Chen and Storey (2006) for a more relaxed threshold. The model also can have other covariates. Moreover, several functions were developed to provide graphical (Fig. 1) and text output. Additionally, interval mapping and inclusive composite interval mapping options (Li et al., 2006) are also included. To illustrate the usage of the package, a simulated dataset from Gazaffi et al. (2014) and its analysis are included in the package, with a full tutorial and vignettes, explaining how to deal with partially informative markers.

With the results of QTL analysis, based on logarithm of the odds scores of hypothesis tests (Gazaffi et al., 2014), users can visualize their genetic effects, statistical significance, segregation patterns and linkage phase with flanking markers. Based on a least square approximation, fullsibQTL can also compute the amount of phenotypic variation explained by each QTL in the model (the coefficient of determination, $R^{2}$ ).

Based on a sound QTL mapping methodology for outcrossing species, and on a reliable multipoint genetic map, provides a QTL mapping toolbox to study the genetic architecture of quantitative traits in outcrossing 


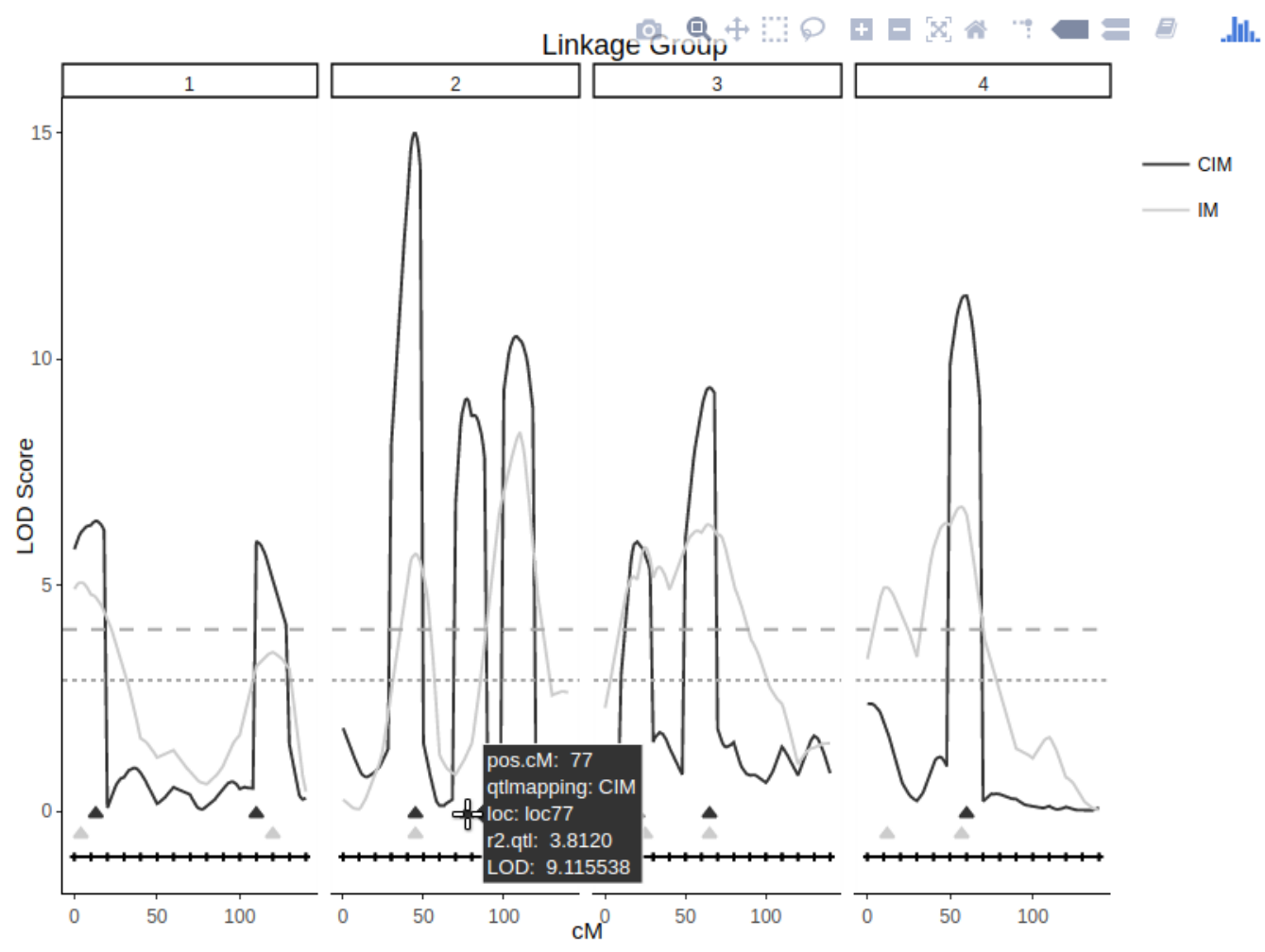

Figure 1. Screenshot of a fullsibQTL interactive HTML+plot ly graphic. It provides a comparison of composite interval mapping and interval mapping approaches for a simulated population. The X-axis shows genetic map for each linkage group, and the y-axis the corresponding logarithm of the odds score for testing the presence of putative QTL. At the top right, there are buttons to zoom, box selection, etc. The mouse hover shows $R^{2}$, position, and logarithm of the odds scores.

62 species. It allows access to relevant information, and includes several graphical and analytical features,

63 providing an ideal environment for interaction with users. Its initial versions were extensively tested and

64 successfully applied several real world problems.

\section{ACKNOWLEDGEMENTS}

65 Authors thank André Conson and Marianella Quezada for their helpfull feedbacks as beta testers, and Karl

66 Broman for sharing code.

\section{FUNDING}

67 This work was supported by the National Council for Scientific and Technological Development - CNPq 68 [grant number 131998/2016-1 to RRA, and 310139/2018-0 for AAFG]; by the Coordination for the

69 Improvement of Higher Education Personnel - CAPES [grant number 3400/2013]; and by São Paulo

70 Research Foundation - FAPESP [grant number TT-5 2009/14068-0 for RG]. 


\section{REFERENCES}

Balsalobre, T. W. A., da Silva Pereira, G., Margarido, G. R. A., Gazaffi, R., Barreto, F. Z., Anoni, C. O., et al. (2017). Gbs-based single dosage markers for linkage and qtl mapping allow gene mining for yield-related traits in sugarcane. BMC Genomics 18, 72. doi:10.1186/s12864-016-3383-x

Barreto, M. A., Rosa, J. R. B. F., Holanda, I. S. A., Cardoso-Silva, C. B., Vildoso, C. I. A., Ahnert, D., et al. (2018). Qtl mapping and identification of corresponding genomic regions for black pod disease resistance to three phytophthora species in theobroma cacao 1. Euphytica 214, 188. doi:10.1007/ s10681-018-2273-5

Broman, K. W., Wu, H., Sen, S., and Churchill, G. A. (2003). R/qtl: Qtl mapping in experimental crosses. Bioinformatics 19, 889-890. doi:10.1093/bioinformatics/btg112

Chen, L. and Storey, J. D. (2006). Relaxed significance criteria for linkage analysis. Genetics 173, 2371-2381. doi:10.1534/genetics.105.052506

Churchill, G. A. and Doerge, R. W. (1994). Empirical Threshold Values for Quantitative Trait Mapping. Genetics 138, 963-971

Conson, A. R., Taniguti, C. H., Amadeu, R. R., Andreotti, I. A., de Souza, L. M., dos Santos, L. H., et al. (2018). High-resolution genetic map and qtl analysis of growth-related traits of hevea brasiliensis cultivated under suboptimal temperature and humidity conditions. Frontiers in Plant Science 9, 1255. doi:10.3389/fpls.2018.01255

Costa, E. A., Anoni, C. O., Mancini, M. C., Santos, F. R. C., Marconi, T. G., Gazaffi, R., et al. (2016). Qtl mapping including codominant snp markers with ploidy level information in a sugarcane progeny. Euphytica 211, 1-16. doi:10.1007/s10681-016-1746-7

Curtolo, M., Granato, L. A.-s. M., Soratto, T. A. T., Curtolo, M., Gazaffi, R., Takita, M. A. A., et al. (2020). Expression Quantitative Trait Loci (eQTL) mapping for callose synthases in intergeneric hybrids of Citrus challenged with the bacteria Candidatus Liberibacter asiaticusenglish. Genetics and Molecular Biology 43

da Silva Pereira, G., Di Cassia Laperuta, L., Nunes, E. S., Chavarría, L., Pastina, M. M., Gazaffi, R., et al. (2017). The sweet passion fruit (passiflora alata) crop: Genetic and phenotypic parameter estimates and qtl mapping for fruit traits. Tropical Plant Biology 10, 18-29. doi:10.1007/s12042-016-9181-4

Gazaffi, R., Margarido, G. R. A., Pastina, M. M., Mollinari, M., and Garcia, A. A. F. (2014). A model for quantitative trait loci mapping, linkage phase, and segregation pattern estimation for a full-sib progeny. Tree Genetics \& Genomes 10, 791-801. doi:10.1007/s11295-013-0664-2

Kao, C.-H., Zeng, Z.-B., and Teasdale, R. D. (1999). Multiple interval mapping for quantitative trait loci. Genetics 152, 1203-1216

Lander, E. S. and Botstein, D. (1989). Mapping mendelian factors underlying quantitative traits using rflp linkage maps. Genetics 121, 185-199

Li, H., Ye, G., and Wang, J. (2006). A modified algorithm for the improvement of composite interval mapping. Genetics

Margarido, G. R. A., Mollinari, M., Broman, K., Ferreira, G. C., Amadeu, R. R., Taniguti, C. H., et al. (2020). onemap: Software for constructing genetic maps in experimental crosses: full-sib, RILs, F2 and backcrosses. R package version 2.1.3

Margarido, G. R. A., Pastina, M. M., Souza, A. P., and Garcia, A. A. F. (2015). Multi-trait multienvironment quantitative trait loci mapping for a sugarcane commercial cross provides insights on the inheritance of important traits. Molecular Breeding 35, 175. doi:10.1007/s11032-015-0366-6

Margarido, G. R. A., Souza, A. P., and Garcia, A. A. F. (2007). Onemap: software for genetic mapping in outcrossing species. Hereditas 144, 78-79. doi:10.1111/j.2007.0018-0661.02000.x 
Meng, L., Li, H., Zhang, L., and Wang, J. (2015). Qtl icimapping: Integrated software for genetic linkage map construction and quantitative trait locus mapping in biparental populations. The Crop Journal 3, 269 - 283. doi:http://dx.doi.org/10.1016/j.cj.2015.01.001. Special Issue: Breeding to Optimize Agriculture in a Changing World

Mollinari, M., Margarido, G. R. A., Vencovsky, R., and Garcia, A. A. F. (2009). Evaluation of algorithms used to order markers on genetic maps. Heredity 103, 494-502. doi:10.1038/hdy.2009.96

R Core Team (2017). R: A Language and Environment for Statistical Computing. R Foundation for Statistical Computing, Vienna, Austria

Soratto, T. A. T., Curtolo, M., Marengo, S., Dezotti, A. L., Lima, R. P. M., Gazaffi, R., et al. (2020). QTL and eQTL mapping associated with host response to Candidatus Liberibacter asiaticus in citrandarins. Tropical Plant Pathology doi:10.1007/s40858-020-00372-7

Souza, L. M., Gazaffi, R., Mantello, C. C., Silva, C. C., Garcia, D., Le Guen, V., et al. (2013). Qtl mapping of growth-related traits in a full-sib family of rubber tree (hevea brasiliensis) evaluated in a sub-tropical climate. PLOS ONE 8, 1-10. doi:10.1371/journal.pone.0061238

Van Ooijen, J. (2004). MapQTL® 5. Software for the mapping of quantitative trait loci in experimental populations. Kyazma BV, Wageningen

Wang, S., Basten, C. J., and Zeng, Z. B. (2012). Windows QTL Cartographer 2.5. Department of Statistics, North Carolina State University, Raleigh, NC

Wu, R., Ma, C.-X., Painter, I., and Zeng, Z.-B. (2002a). Simultaneous maximum likelihood estimation of linkage and linkage phases in outcrossing species. Theoretical Population Biology 61, 349 - 363. doi:http://dx.doi.org/10.1006/tpbi.2002.1577

Wu, R., Ma, C. X., Wu, S. S., and Zeng, Z. B. (2002b). Linkage mapping of sex-specific differences. Genetical Research 79, 85-96. doi:10.1017/S0016672301005389

Zeng, Z. B. (1993). Theoretical basis for separation of multiple linked gene effects in mapping quantitative trait loci. Proceedings of the National Academy of Sciences 90, 10972-10976

Zeng, Z. B. (1994). Precision mapping of quantitative trait loci. Genetics 136, 1457-1468 